\title{
Dietary considerations for the sick and hospitalized patients
}

\author{
S Kumari ${ }^{1}, \mathrm{M} \mathrm{Vikram}^{2}$ \\ Department of Nutrition and Dietetics ${ }^{1}$, Department of Conservative Dentistry and Endodontics ${ }^{2}$ \\ B.P. Koirala Institute of Health Sciences, Dharan, Nepal
}

\begin{abstract}
From a clinical perspective, knowledge of the nutritional status of a hospitalized patient is an indispensable strategy for establishing an adequate approach to the maintenance and/or recovery of nutritional status during the hospital stay. Nutritional status has an important impact on the course of underlying diseases. Malnutrition increases the rate of morbidity, length of hospital stay and certain other complications. Therefore malnutrition becomes very common among hospitalized patients which in turn lead to morbidity and increased hospital stay. Nutritional requirement of the patient vary in different disease condition and their mode of feeding depends upon the condition of the patient and the functioning organ.
\end{abstract}

Keywords: Hospital stay, malnutrition, nutrition

\section{Introduction}

For a healthy living the diet should provide adequate amounts of all the nutrients. A balanced diet is one that contains all the nutrients in right proportion. The proportion of protein, fat and carbohydrate is approximately 1:1:4. Nutritional assessment reveals the nutritional status of a patient. It thereby helps identify each patient's need for specific nutritional care and facilitates early intervention. ${ }^{1}$

\footnotetext{
Address for correspondence

Shilpi Kumari

Dietitian

Department of Nutrition and Dietetics

B.P. Koirala Institute of Health Sciences, Dharan

E-mail: shilpi.dietitian@gmail.com
}

Globally, malnutrition is an important problem but often under-recognized in the hospital population. Many patients presenting to hospitals are malnourished, and some do develop a degree of iatrogenic malnutrition, due to repeated fasting for tests and procedures, and a lack of nutritional knowledge by hospital staff. ${ }^{2}$

Malnutrition is related with a higher susceptibility to infections, due in part to a decrease in cell immunity, a reduction of secretary $\lg \mathrm{A}$, a decrease in alveolar macrophage function, and a growth of bacterial colonization and adhesion in the upper and lower airways. ${ }^{3}$ 
Malnutrition interferes with the appropriate response of the body to disease and predisposes to infection and to the onset of multi organ failure, increasing morbidity and mortality, the mean length of hospital stay, and health costs. ${ }^{4}$ Malnutrition is not limited to the general ward patients. Intensive care patients are often malnourished, which can result in increased infectious complications, poor wound healing, prolonged ventilation dependence with increased morbidity and mortality. ${ }^{2}$

Percutaneous endoscopic gastrostomy (PEG) has become an established procedure for enteral feeding of patients. The aim of the procedure is to avoid further weight loss and to increase or rather stabilize the patient's weight. Despite the fact that this method is simple and quick, it is still associated with a significantly high level of early mortality. ${ }^{5}$

A percutaneous endoscopic gastrostomy (PEG) is used as an alternative feeding method in patients treated in intensive care, neurology, and reanimation clinics who cannot be adequately fed orally. An enteral feeding method is ideal for patients who cannot be fed orally but who have normal gastrointestinal functions. ${ }^{6}$

Enteral feeding can be achieved with a nasogastric, nasojejunal, gastrostomy, or jejunostomy tube. For long-term feeding, gastrostomy and jejunostomy tubes are generally preferred. Today, one of the most common methods is a percutaneous gastrostomy tube.

\section{Nutritional requirements}

For the malnourished patient, actual weight is used to calculate required calories, whereas in the obese patient, the patient's ideal weight should be used. To calculate the basal energy expenditure, many use the HarrisBenedict equation ${ }^{11}$ (Table 1).

\section{Table 1: Harris benedict equation (Basal energy expenditure - BEE)}

\begin{tabular}{|l|}
\hline Men: $66+(13.7 \times$ weight $)+(5 \times$ height $)-(6.8 \times$ \\
age) \\
\hline Women: $655+(9.6 \times$ weight $)+(1.8 \times$ height $)-$ \\
$(4.7 \times$ age $)$ \\
\hline $\begin{array}{l}\text { Variables: gender, weight }(\mathrm{kg}) \text {, height }(\mathrm{cm}), \text { age } \\
\text { (years) }\end{array}$ \\
\hline
\end{tabular}

Once the basal energy expenditure has been calculated, the calorie requirements can be calculated factoring in the activity and stress factor (Table 2 and Table 3 ). If the calculations seem quite complex, then the quick rule of thumb would be, each patient requires $25-30 \mathrm{kcal}$ per kilogram of weight per day. ${ }^{2}$ 
Table 2: Formulae for calculation of daily calorie requirement

Daily calorie requirement $=\mathrm{BEE} \times$ activity

factor $x$ stress factor

Table 3: Conditions and stress factor levels

\begin{tabular}{|c|l|}
\hline Injury & Stress factor \\
Minor surgery & 1.0 to 1.1 \\
Long bone fracture & 1.15 to 1.3 \\
Cancer & 1.1 to 1.3 \\
Peritonitis / sepsis & 1.1 to 1.3 \\
Severe infection / multi & 1.2 to 1.4 \\
trauma & 1.2 to 1.4 \\
Multi-organ failure & 1.2 to 2.0 \\
Burns & \\
Activity & 1.2 \\
Confined to bed \\
Out of bed
\end{tabular}

Nutrient requirement is defined as the minimum amount of the absorbed nutrients that is necessary for maintaining the normal physiological functions of the body. A balanced diet is one which contains different types of foods in such quantity and proportion so that the need for calorie, protein, minerals, vitamins and other nutrients is adequately met and a small provision is made for extra nutrients to withstand the duration of illness. In addition balanced diet should provide bioactive phytochemicals such as dietary fiber, antioxidant and nutraceuticals which have positive health benefits. ${ }^{7}$ Planning of balance diet should aim that the diet contains various groups of food stuffs such as energy yielding, body building and protective foods in the right proportion.

A balanced diet provides about 60-65\% carbohydrate, $20-25 \%$ fat and $10-15 \%$ proteins. In a patient who is catabolic, the ratios differ. A minimum of $100 \mathrm{gm}$ per day of carbohydrates are required to prevent ketosis. When giving lipids, they are used both for energy requirements but also as a source of essential fatty acids such as linoleic acid. Increased protein is required to help prevent muscle loss due to catabolism. ${ }^{2}$

The nutritional care of the hospital patient involves the co-ordinated team approach of the doctor, dietitian and nurses (Fig 1). ${ }^{8}$

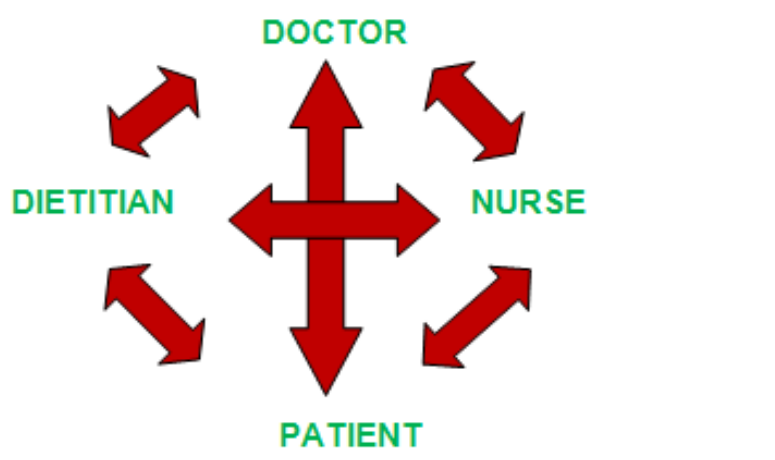

Fig 1. Channels of diet counseling between patient, doctor, dietitian \& nurses

The above team should take the burden of educating the patients and their attendants about the diet in curing the disease and its ill effects if not following the prescribed diet. 


\section{Malnutrition in patient}

It is well known that nutritional status is a major determinant of the health and wellbeing and there is no doubt regarding the importance of the studies of nutritional status on patients according to spatial and temporal dimension. In developing countries, children and adults are vulnerable to malnutrition because of low dietary intakes, infectious diseases, lack of appropriate care, and inequitable distribution of food within the household. $^{9}$

Diseases like cancers, dementia, chronic infectious diseases, tuberculosis and any end stage organ disease leads to decreased nutritional state. Most of these conditions results in reduced oral intake either through reduced appetite, disordered swallowing or digestion or catabolic/cachexia state. Hence malnutrition detection is important for evaluation of the exact incidence and further nutritional management. Many illnesses caused by the infection, injuries and metabolic disorders affect the food intake of the patients leading to malnutrition and undernutrition. $^{2,8}$ Early detection of malnutrition in hospitalized patients and nutritional intervention can result in decreased morbidity such as decreased pneumonia and infections and decreased length of hospital stay.

Often in clinical practice, a simplified assessment of the patient's nutritional state is necessary. A patient with temporal muscle wasting, unintentional weight loss and/or decreased intake pre-hospital admission, with a disease state is at risk of malnutrition and patient will usually be at least moderately malnourished. ${ }^{2}$

There is a need of community based recommendations for nutrition and social science approaches, in the prevention of chronic diseases. This could be achieved by scientists with high standards of tertiary education in several medical and paramedical sciences and professions, as internal medicine, nutrition-dietetics, nursing and midwifery. $^{10}$

A standardized schedule should be established for the advancement of enteral and oral nutrition in the hospital. The speed of parentral nutrition reduction depends on the tolerance of enteral or oral nutrition. Based on expert opinion, nutrition consultation during the phase of advancing enteral and oral nutrition is useful, particularly in patients who received parentral nutrition for more than 14 days and in patients with gastroenterological disease. ${ }^{13}$

\section{Mode of feeding}

While planning a diet for the patient his socioeconomic status, dietary and medical history is to be considered. Oral feeding is the best way to ensure the nourishment of a patient. It is only when a patient is unable to take oral 
feeds that enteral tube feeding or intravenous feeding should be used. $2,7,8,11$

Enteral nutrition can be provided either orally or by tube feeding. By definition enteral means "within or by the way of the gastrointestinal tract". This is done by passing a tube into the stomach or jejunum through the nose which is called nasogastric feeding. ${ }^{7}$ Enteral alimentation is the preferred modality of support for seriously ill patients who have acceptable gastrointestinal function but are unable to maintain oral diet. Advantages of enteral feeding include enhancement of intestinal mucosal integrity and nutrient absorption, improvement of metabolic and immune response, and reduction of costs and further complications. ${ }^{4,5,12}$

When a patient requires prolonged tube feeding (greater than 30 days), a tube gastrostomy or enterostomy is considered. Nutrition supplied through the tube may be: a) natural liquid foods; b) blenderised foods; or c) commercially supplied elemental diets ${ }^{11}$. Sometimes when the nutritional requirement is not fulfilled by the diet certain supplements (powder products) are added to make it nutritionally adequate. Nutritional supplements are disease specific, but these supplements are expensive; out of the reach of poor patient. In such conditions use of normal blenderised feed with the incorporation of high calorie and protein foods like ice-cream, eggs, butter etc can be used as the condition requires.

In patients who are unable to eat, for example stroke patients, consideration for nasogastric tube feeding is important. If there is a high risk of reflux and aspiration or if high aspirates are obtained from the nasogastric tube, and yet the gastrointestinal tract is functional then a naso-enteric tube can be considered for feeding. If the patient requires long term feeding via nasogastric or nasoenteric tube then insertion of a percutaneous endoscopic gastrostomy (PEG) or jejunostomy (PEJ) tube may be appropriate. $^{2}$ The literature also supports tube feeding via nasogastric tube or percutaneous endoscopic gastrostomy (PEG) as a means of minimizing loss of weight in patients. ${ }^{14}$

Although the preferred route of providing the nutritional support to the patient is enteral most of the enteral nutrition alone cannot meet the nutritional requirement of patients. So, a combined supplemental parenteral and enteral feeding is given to meet the energy and protein requirements. ${ }^{15}$

Intravenous feeding requires a team approach by the clinician, dietitian, pharmacist and nurses. ${ }^{2,11}$ Intravenous feeding is prohibitively expensive, requires constant laboratory surveillance and expert know-how, and is associated with complication. Total parentral nutrition for 
more than three months may produce painful bone diseases. Intravenous feeding for more than a month produces falling of hair and depression. ${ }^{11}$ Therefore intravenous feeding is never considered if the patient can be fed orally or through enteral route.

\section{Conclusion}

Malnutrition is a comprehensive term. Malnutrition in a patient can be before admission to a hospital or during the hospital stay. Malnutrition can be corrected through proper nutrition. So, nutritional awareness and knowledge among the hospital staff is important for treating a nutritionally depleted patient. Maintenance of nutritional status is important, which can be done either through oral or enteral or parentral feeding.

\section{References}

1. Tienboon P. Nutrition problems of hospitalized children in a developing country. Asia Pacific Journal of Clinical Nutrition, 2002; 11 (4), 258-62.

2. Felicia E, Eeshen J. Nutrition in the hospital setting. Brunei Int Med J. 2012; 8 (1): 12-18.

3. Martins FI. Chronic obstructive pulmonary disease and malnutrition: why are we not winning this battle? Jornal de Pneumologia 2003; 29(2):107-115
4. Prieto MB, Cid JLH. Malnutrition in the critically III Child: The importance of enteral nutrition. Int $\mathrm{J}$ Environ Res Public Health 2011; 8: 4353-66

5. Zopf Y, Maiss J, Konturek P, Rabe C, Hahn EG and Schwab D. Predictive factors of mortality after PEG insertion: Guidance for clinical practice. Journal of Parenteral and Enteral Nutrition 2011; $35: 50-55$

6. Çakır M, Tekin A, Küçükkartalla $T$, Vatansev C, Aksoy F, Kartal A. Dergisi DT. Long-term results of percutaneous endoscopic gastrostomies, Dicle Medical Journal 2012; 39 (2): 162-165.

7. Srilakshmi B. Dietetics. $5^{\text {th }}$ ed. New Age International Pvt Ltd. New Delhi; 2006.

8. Swaminathan M. Essentials of food and nutrition. Vol 2, $2^{\text {nd }}$ ed. The Banglore printing and publication Co. Itd. (1995)

9. Luxmi Y, Sachdeva M P. Prevalence of malnutrition among the Chiru children of Manipur, India. International Journal of Human Science 2012; (9)2, 405-16

10. Fragkiadakis $G$ A, Papandreou $C$ and Gkouskou K Nutrition in Tertiary education: Biological and social approaches $\mathrm{J}$. Social Sci 2012; 8 (2): 120-25.

11. Antia FP, Abraham P. Clinical dietetics and nutrition. $4^{\text {th }}$ ed. Oxford university press, 2005.

12. Serpa LF, Kimura M, Faintuch J, Ceconello I. Effects of continuous 
versus bolus infusion of enteral nutrition in critical patients.2003 Rev. Hosp. Clin. 2003; 58 (1): 9-14.

13. Bischoff S C, Kester L, Meier R, Radziwill R, Schwab D, Thul P. Organization, regulations, preparation and logistics of parenteral nutrition in hospitals and homes; the role of the nutrition support team - Guidelines on Parenteral Nutrition. GMS German Medical Science 2009; 7Doc20.

14. Jeffery E, Sherriff J, Langdon C. A clinical audit of the nutritional status and need for nutrition support amongst head and neck cancer patients treated with radiotherapy. Australasian Medical Journal 2012; 5(1): 8-13

15. Hsu MH, Yu YE, Tsai YM, Lee HC, Huang YC, Hsu HS. Combined enteral feeding and total parenteral nutrition support improves outcome in surgical intensive care unit patients. Journal of Chinese Medical Association 2012; 75(9):459-63. 